\title{
6,5-Fused Ring, C2-Salvinorin Ester, Dual Kappa and Mu Opioid Receptor Agonists as Analgesics Devoid of Anxiogenic Effects
}

Nicholas S. Akins, ${ }^{1}$ Nisha Mishra, ${ }^{1}$ Hannah M. Harris, ${ }^{2}$ Narendar Dudhipala, ${ }^{3}$ Seong Jong Kim, ${ }^{4}$ Adam W. Keasling, ${ }^{1}$ Soumyajit Majumdar, ${ }^{2,3}$ Jordan K. Zjawiony, ${ }^{1,2}$ Nicole M. Ashpole, ${ }^{1,2}$ Hoang V. Le $\mathrm{e}^{1,2^{*}}$

${ }^{1}$ Department of BioMolecular Sciences, School of Pharmacy, University of Mississippi, University, Mississippi 38677, U.S.A.

${ }^{2}$ Research Institute of Pharmaceutical Sciences, School of Pharmacy, University of Mississippi, University, Mississippi 38677, U.S.A.

${ }^{3}$ Department of Pharmaceutics and Drug Delivery, School of Pharmacy, University of Mississippi, University, Mississippi 38677, U.S.A.

${ }^{4}$ Natural Products Utilization Research Unit, United States Department of Agriculture, Agricultural Research Service, University, MS 38677, U.S.A.

ABSTRACT: Analgesia is commonly mediated through the mu or kappa opioid receptor agonism. Unfortunately, selective mu or kappa receptor agonists often cause harmful side effects. Recently, ligands exhibiting dual agonism to the opioid receptors, such as to mu and kappa, or to mu and delta, have been suggested to temper undesirable adverse effects while retaining analgesic activity. Herein we report an introduction of various 6,5-fused rings to $\mathrm{C} 2$ of the salvinorin scaffold via an ester linker. In vitro studies showed that some of these compounds have dual agonism on kappa and mu opioid receptors, while some have triple agonism on kappa, mu, and delta. In vivo studies on the lead dual kappa and mu opioid receptor agonist, compound 10, showed that it produced analgesic activity while avoiding anxiogenic effects in murine models, thus providing further strong evidence for the therapeutic advantages of dual opioid receptor agonists over selective opioid receptor agonists.

KEYWORDS: 6,5-Fused ring, salvinorin, dual kappa and mu opioid receptor agonists, analgesics, no anxiogenic effects

The Centers for Disease Control (CDC) has estimated that one in five Americans suffers from chronic pain, and one in six American adults suffers from a mental health disorder. ${ }^{1,2}$ Studies have indicated these disorders share a neural network and thus have a bidirectional relationship. ${ }^{1}$ Therefore, discovery and development of new analgesics that exert efficacy for pain and mental health disorders are vital. Historically, selective agonists of classical opioid receptors, including kappa $(\kappa, \mathrm{KOR}), \mathrm{mu}(\mu$, MOR), and delta opioid receptor $(\delta, D O R)$, are commonly used and clinically efficacious against states. ${ }^{1} \quad$ Unfortunately, these compounds often result in many serious and harmful adverse effects, such as addiction, dysphoria, reduced bowel motility, tolerance, convulsions, and overdose. $^{3,4}$ The opioid epidemic has thus sparked an urgent need to develop effective pain management therapeutics devoid of such adverse effects. $^{3}$ Recently, compounds exhibiting dual agonism to the opioid receptors, such as to MOR and $\mathrm{KOR},{ }^{5,6}$ or to MOR and DOR, ${ }^{7}$ have been suggested to temper undesirable adverse effects while retaining analgesic activity. 


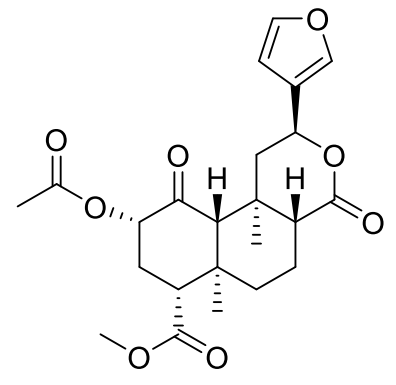

salvinorin A

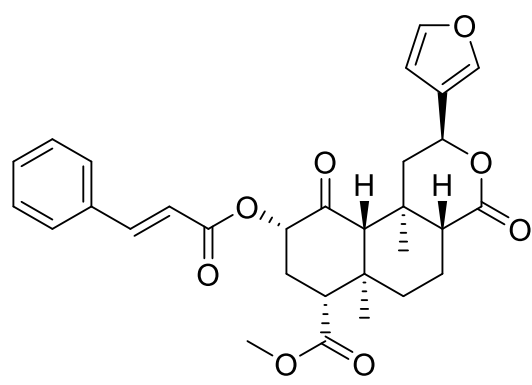

PR-38

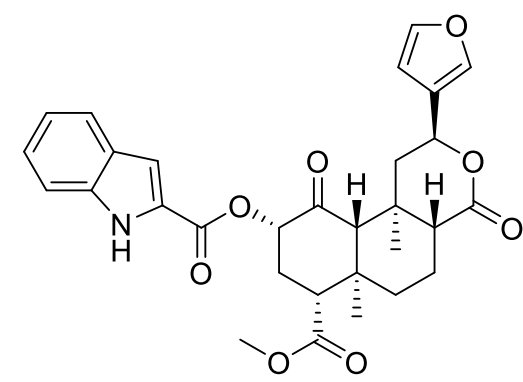

salvindolin

Figure 1: Structures of salvinorin A, PR-38, and salvindolin.

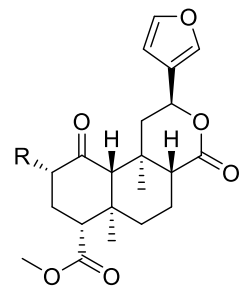

1. $R=$

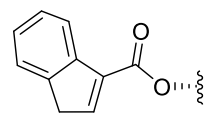

2. $R=$

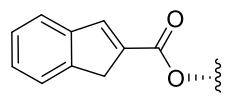

3. $R=$

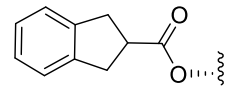

4. $R=$

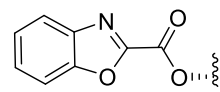

9. $\mathbf{R}=$
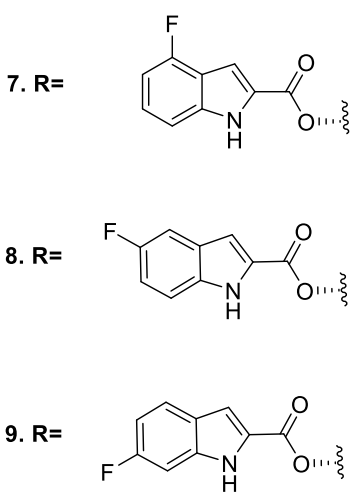

12. $R$

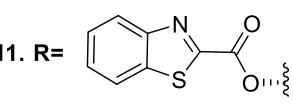

7. $R=$<smiles>[R]=[Pt]=Cc1ccc2[nH]c(C(=O)OC)nc2c1</smiles>

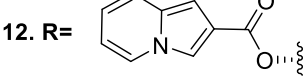

5. $R=$

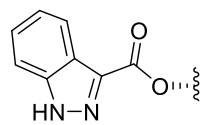

10. $R=$

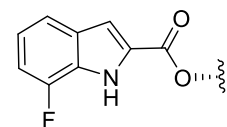

14. $R=$

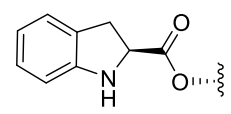

Figure 2: Introduction of various 6,5-fused rings to $\mathrm{C} 2$ of the salvinorin scaffold via an ester linker: structures of compounds 1-14.

Salvinorin A (Figure 1) is the main active ingredient in the hallucinogenic plant Salvia divinorum. It is one of the most potent, naturally occurring opioid agonists with high selectivity and affinity for KOR. ${ }^{8}$ It has the potential to be beneficial in therapy of various central nervous system (CNS) disorders. Salvinorin A induces analgesic and anti-inflammatory effects, but uniquely does not present affinity to other receptors associated with perceptual alterations, such as dopamine, serotonin, or glutamate receptors. ${ }^{8}$ Due to its strong hallucinating effects, salvinorin A has never advanced to clinical trials, ${ }^{9}$ but has been used as an important prototype for the development of related drug candidates, especially salvinorin analogs that exert therapeutic effects while being devoid of the hallucinatory side effects of KOR agonists. ${ }^{10-12}$

Recently, salvinorin analogs with aromatic or heteroaromatic moieties at $\mathrm{C} 2$ have been shown to exhibit significant changes in pharmacological profile, associated with a change in the affinity from KOR to MOR. ${ }^{5,6}$ Two representative molecules of this change are PR-38 and salvindolin (Figure 1), both of which display dual affinity to KOR and MOR. ${ }^{5,6}$ PR-38 had roughly a five-fold preference towards KOR, while salvindolin displayed a 100-fold preference towards MOR. 
A)

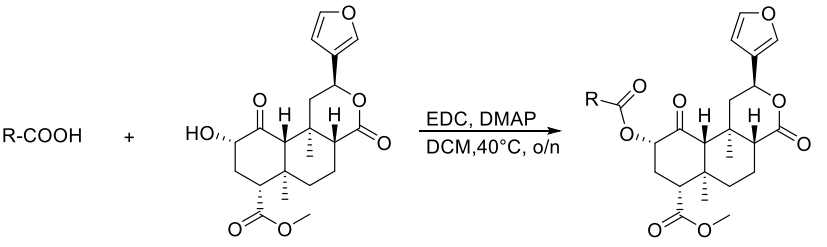

salvinorin B

1:1H-indene-3-, Yield: 49\%

2:1H-indene-2-, Yield: $66 \%$

3:1H-indane-2-, Yield: $85 \%$

4: Benzo[d]oxazole-2-, Yield: $25 \%$

5: $1 \mathrm{H}$-indazole-3-, Yield: $25 \%$

6: 1 H-benzo[d]imidazole-2-, Yield: $42 \%$

7: 4-fluoro-1H-indole-2-, Yield: $42 \%$

8: 5 -fluoro-1H-indole-2-, Yield: $43 \%$

9: 6 -fluoro-1H-indole-2-, Yield: $49 \%$

10: 7 -fluoro- $1 \mathrm{H}$-indole-2-, Yield: $41 \%$

B)

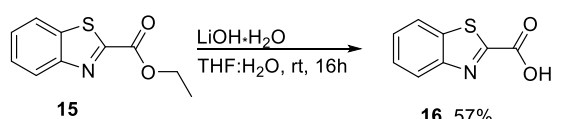

15

$16,57 \%$
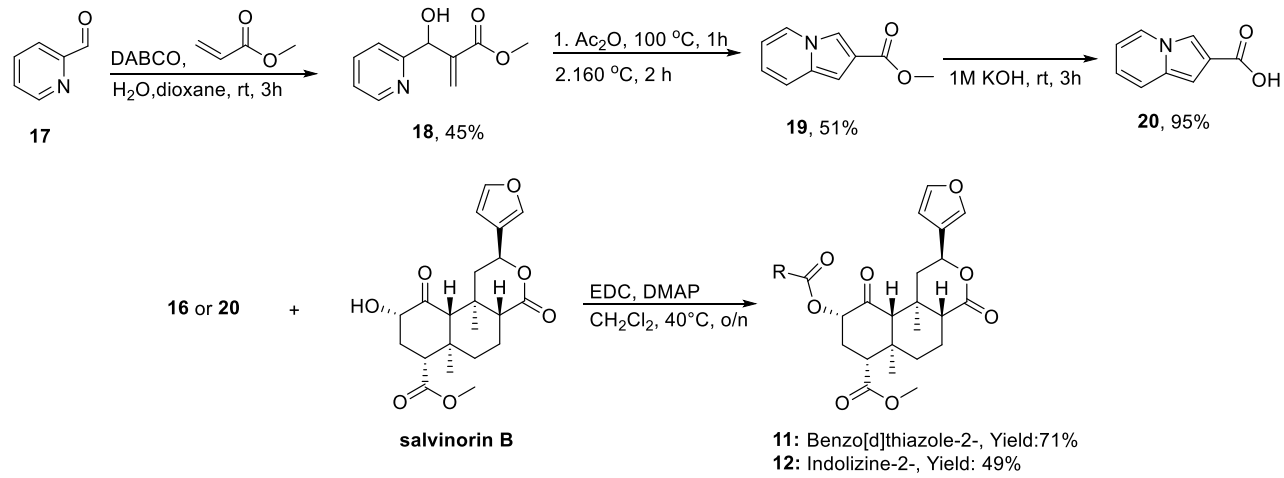

C)

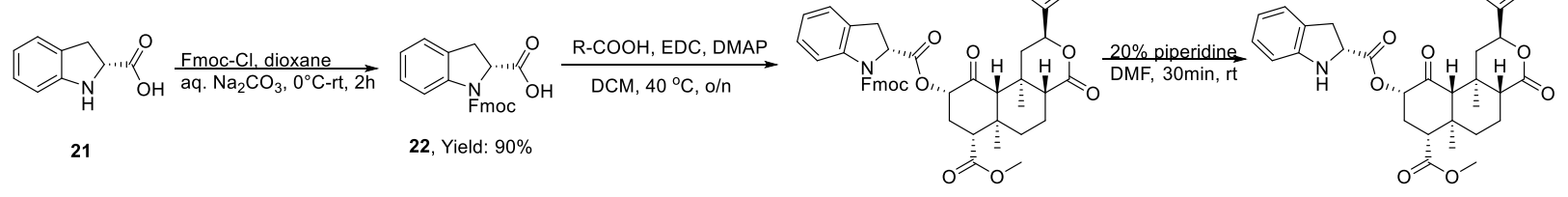

23: (R)-1-(((Fmoc)methoxy)carbonyl)indoline-2-, Yield: 89\%

13: (R)-1-H-indoline-2-, Yield: $84 \%$

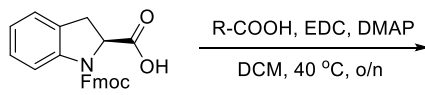

24

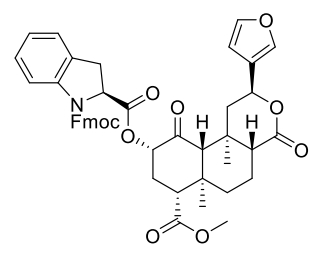

25: (S)-1-(((Fmoc)methoxy)carbonyl)indoline-2-,Yield: 71\%

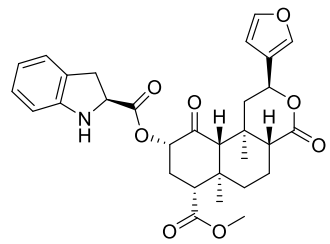

14: (S)-1-H-indoline-2-, Yield: $75 \%$

Scheme 1: Synthesis of compounds 1-10 (A), 11-12 (B), and 13-14 (C)

To study the mechanism for the switch to dual affinity and the effects of novel moieties at C2, we introduced a series of 6,5-fused rings to $\mathrm{C} 2$ via an ester linker (Figure 2), including the indene (which is non-nitrogenous, compounds $\mathbf{1}$ and 2), the indane (which is non-aromatic, compound $\mathbf{3}$ ), and several bicyclic heterocycles (other compounds). We planned to use these synthetic salvinorin analogs to study the structure-activity relationship between variation of the 6,5-fused rings and the KOR, MOR, and DOR.

Compounds 1-10 were synthesized via an ester coupling reaction between commercially available salvinorin $\mathrm{B}$ and the corresponding carboxylic acids, which were also commercially available, using EDC and DMAP, ${ }^{19}$ in various yields (Scheme 1A). 
Table 1. $K \mathrm{i}$ and $\mathrm{EC}_{50}$ values of compounds 1-14 towards KOR, MOR, and DOR

\begin{tabular}{|c|c|c|c|c|c|c|c|}
\hline Compound & $C(2)$ substituent & $\begin{array}{l}\mathbf{K}_{\mathrm{i}}, \mathbf{n M} \\
(\mathbf{K O R})\end{array}$ & $\begin{array}{l}\mathrm{EC}_{50}, \mathrm{nM} \\
(\mathrm{KOR})\end{array}$ & $\mathrm{K}_{\mathrm{i}}, \mathrm{nM}$ (MOR) & $\begin{array}{l}\mathbf{E C}_{50}, \mathbf{n M} \\
(\mathbf{M O R})\end{array}$ & $\begin{array}{l}\mathrm{K}_{\mathrm{i}}, \mathbf{n M} \\
(\mathrm{DOR})\end{array}$ & $\begin{array}{l}\mathrm{EC}_{50,}, \mathrm{nM} \\
\text { (DOR) }\end{array}$ \\
\hline Naloxone & $\overline{--}$ & $9.98 \pm 1.69$ & & $4.39 \pm 0.68$ & & $2.09 \pm 0.32$ & \\
\hline U69,593 & -- & -- & $126.3 \pm 12.9^{\mathrm{c}}$ & -- & -- & -- & -- \\
\hline DPDPE & -- & -- & -- & -- & -- & & \\
\hline Morphine & -- & -- & -- & & 416.1 & -- & -- \\
\hline Cebranopadol & -- & $3.77 \pm 0.67$ & NT & $3.77 \pm 0.67$ & NT & & NT \\
\hline Nalbuphine & -- & $4.02 \pm 0.64$ & NT & $4.02 \pm 0.64$ & NT & & NT \\
\hline Naltrexone & -- & $12.9 \pm 2.26$ & NT & $12.9 \pm 2.26$ & NT & & NT \\
\hline Salvindolin & 1H-Indole-2- & $1005 \pm 169^{\mathrm{e}}$ & NT & $10.49 \pm 1.79^{\mathrm{e}}$ & $187^{\mathrm{e}}$ & & \\
\hline 1 & 1H-Indene-3- & $62.3 \pm 12.6$ & $1570 \pm 643^{\mathrm{d}}$ & $531.4 \pm 219.3$ & $>10,000^{\mathrm{b}}$ & $\mathrm{Nd}^{\mathrm{a}}$ & \\
\hline 2 & 1H-Indene-2- & $49.7 \pm 15.5$ & $853 \pm 230^{c}$ & $538.1 \pm 113.3$ & $49.5 \pm 5.65^{\mathrm{c}}$ & $35.25 \pm 5.7$ & $2347 \pm 845^{\mathrm{d}}$ \\
\hline 3 & 1H-Indane-2- & $1265 \pm 269$ & $2998 \pm 1868^{\mathrm{c}}$ & $3015 \pm 1342$ & $>10,000^{\mathrm{b}}$ & $\mathrm{Nd}^{\mathrm{a}}$ & $\mathrm{Nd}^{\mathrm{a}}$ \\
\hline 4 & Benzo[d]oxazole-2- & $\mathrm{Nd}^{\mathrm{a}}$ & $\mathrm{Nd}^{\mathrm{a}}$ & $\mathrm{Nd}^{\mathrm{a}}$ & -- & $\mathrm{Nd}^{\mathrm{a}}$ & $\mathrm{Nd}^{\mathrm{a}}$ \\
\hline 5 & 1H-indazole-3- & $\mathrm{Nd}^{\mathrm{a}}$ & $\mathrm{Nd}^{\mathrm{a}}$ & $207 \pm 61.0$ & $>10,000^{\mathrm{b}}$ & $\mathrm{Nd}^{\mathrm{a}}$ & $\mathrm{Nd}^{\mathrm{a}}$ \\
\hline 6 & 1H-benzimidazole-2- & $\mathrm{Nd}^{\mathrm{a}}$ & $\mathrm{Nd}^{\mathrm{a}}$ & $83.16 \pm 13.8$ & $>10,000^{\mathrm{b}}$ & $\mathrm{Nd}^{\mathrm{a}}$ & $\mathrm{Nd}^{\mathrm{a}}$ \\
\hline 7 & 4-fluoro-1H-indole-2- & $57.3 \pm 15.3$ & $877 \pm 174^{\mathrm{c}}$ & $13.13 \pm 2.8$ & $211 \pm 86^{\mathrm{d}}$ & $\mathrm{Nd}^{\mathrm{a}}$ & $\mathrm{Nd}^{\mathrm{a}}$ \\
\hline 8 & 5-fluoro-1H-indole-2- & $97.8 \pm 12.8$ & $1110 \pm 411^{\mathrm{d}}$ & $\mathrm{Nd}^{\mathrm{a}}$ & $\mathrm{Nd}^{\mathrm{a}}$ & $\mathrm{Nd}^{\mathrm{a}}$ & $\mathrm{Nd}^{\mathrm{a}}$ \\
\hline 9 & 6-fluoro-1H-indole-2- & $17.0 \pm 3.1$ & $419 \pm 102^{\mathrm{c}}$ & $39.7 \pm 14.6$ & $972 \pm 417.9^{\mathrm{d}}$ & $\mathrm{Nd}^{\mathrm{a}}$ & $\mathrm{Nd}^{\mathrm{a}}$ \\
\hline 10 & 7-fluoro-1H-indole-2- & $53.8 \pm 9.8$ & $36.6 \pm 21.5^{\mathrm{c}}$ & $58.4 \pm 19.3$ & $132 \pm 54^{\mathrm{d}}$ & $\mathrm{Nd}^{\mathrm{a}}$ & $\mathrm{Nd}^{\mathrm{a}}$ \\
\hline 11 & Benzo[d]thiazole-2- & $91.7 \pm 16.5$ & $294 \pm 65^{\mathrm{c}}$ & $\mathrm{Nd}^{\mathrm{a}}$ & ND & $\mathrm{Nd}^{\mathrm{a}}$ & $\mathrm{Nd}^{\mathrm{a}}$ \\
\hline 12 & Indolizine-2- & $18.4 \pm 2.0$ & $1563 \pm 1006^{\mathrm{d}}$ & $1392 \pm 345$ & $200 \pm 43^{\mathrm{d}}$ & $353.1 \pm 66.4$ & $742 \pm 343^{\mathrm{d}}$ \\
\hline 13 & (R)-1-H-indoline-2- & $129.8 \pm 37.3$ & $360.6 \pm 80.4^{\mathrm{d}}$ & $9.04 \pm 1.46$ & $840 \pm 180^{c}$ & $\mathrm{Nd}^{\mathrm{a}}$ & $\mathrm{Nd}^{\mathrm{a}}$ \\
\hline 14 & $(S)-1-\mathrm{H}$-indoline-2- & $32.1 \pm 8.8$ & $2258 \pm 507^{\mathrm{c}}$ & $77.3 \pm 33.2$ & $>10,000^{\mathrm{b}}$ & $\mathrm{Nd}^{\mathrm{a}}$ & $\mathrm{Nd}^{\mathrm{a}}$ \\
\hline \multicolumn{8}{|c|}{$\begin{array}{l}\text { NT Not tested } \\
\mathrm{Nd}^{\mathrm{a}} \text { Not determined; initial screening resulted in an out-of-range value and therefore wasn't tested further } \\
{ }^{\mathrm{b}} \mathrm{EC}_{50} \text { values assays were carried out and the values were above the upper limit of the range }(10,000 \mathrm{nM}) \\
{ }^{\mathrm{c}} \text { Full agonist } \\
{ }^{\mathrm{d}} \text { Partial agonist } \\
{ }^{\mathrm{e}} \text { Literature values }\end{array}$} \\
\hline
\end{tabular}

The corresponding carboxylic acids for compounds 11-14 were not commercially available, so we synthesized them in our lab. A base hydrolysis of the ethyl ester in commercially available 15 using lithium hydroxide provided carboxylic acid $\mathbf{1 6}$ in a 57\% yield (Scheme 1B). An ester coupling reaction between salvinorin B and $\mathbf{1 6}$ gave compound $\mathbf{1 1}$ in $71 \%$ yield. A literature 3-step procedure $^{16}$ to synthesize carboxylic acid $\mathbf{2 0}$ from commercially available $\mathbf{1 7}$ was followed, and the overall yield of the three steps was 22\% (Scheme 1B). An ester coupling reaction between salvinorin $\mathrm{B}$ and $\mathbf{1 7}$ gave compound $\mathbf{1 2}$ in $49 \%$ yield.

A direct ester coupling reaction between salvinorin B and commercially available carboxylic acid $\mathbf{2 1}$ did not yield $\mathbf{1 3}$. Therefore, the free indoline amino group of $\mathbf{2 1}$ was then first protected using the fluorenylmethyloxycarbonyl (Fmoc) group to provide 22 in a 90\% yield (Scheme 1C). An ester coupling reaction between salvinorin B and $\mathbf{1 7}$ gave Fmoc-protected ester $\mathbf{2 3}$ in $89 \%$ yield. A subsequent Fmoc deprotection using $20 \%$ piperidine in DMF ${ }^{13}$ gave compound 13 in $84 \%$ yield. In a similar fashion, an ester coupling reaction between salvinorin $\mathrm{B}$ and commercially available Fmoc-protected carboxylic acid 24, followed by Fmoc deprotection, gave compound $\mathbf{1 4}$ in a 53\% yield over two steps (Scheme 1C).

Compounds 1-14 were subjected to in vitro evaluation to determine their affinity, efficacy, and functionality on classical opioid receptors. A competitive binding assay ${ }^{18}$ was utilized to screen the receptor binding and determine the $K_{\mathrm{i}}$ values of each compound against KOR, MOR, and DOR. These $K_{\mathrm{i}}$ values are shown in Table 1. A GTP $\gamma \mathrm{S}$ assay was also completed to determine the functionality and potency of selected compounds of interest. All of these compounds were shown to be agonists of the opioid receptors, and their $\mathrm{EC}_{50}$ values are shown in Table 1. 


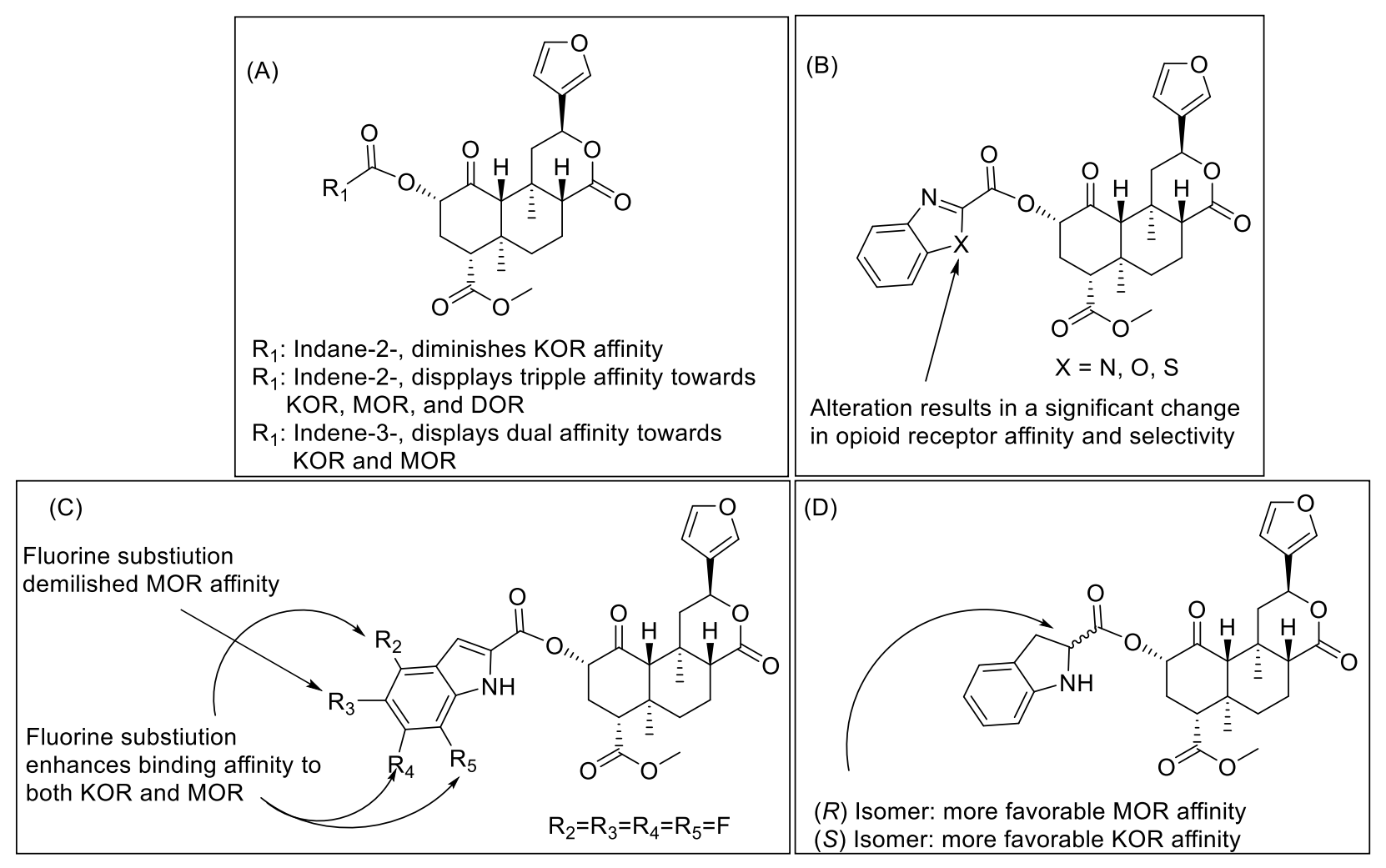

Figure 3: Structure-activity relationship of 6,5-fused rings on the binding affinity towards opioid receptors: (A) non-nitrogenous 6,5-fused rings; (B) 6,5-fused rings that have an additional heteroatom besides nitrogen; (C) fluorine substitutions on the indole ring; (D) breaking the trigonal planar geometry of carbon 2 on the indole ring

It has been hypothesized from the studies of herkinorin, PR-38, and salvindolin that the introduction of an aromatic moiety to $\mathrm{C} 2$ of the salvinorin scaffold substantially changed the pharmacological profile of the compounds from mono affinity (KOR) to dual affinity (KOR and MOR), ${ }^{5,20}$ and that the nitrogen on the indole ring of salvindolin was responsible for the affinity change to MOR by interacting with nearby residues (coming from a molecular modeling study). ${ }^{6}$ However, our results showed that compounds $3, \mathbf{1 3}$, and $\mathbf{1 4}$ do not have an aromatic moiety to $\mathrm{C} 2$ of the salvinorin scaffold and yet exhibited dual activity on KOR and MOR, as well as compounds $\mathbf{1}$ and $\mathbf{2}$ lacking of the nitrogen in the 6,5-fused ring, but still exhibited dual affinity to KOR and MOR. Our results have disproved the aforementioned hypotheses and suggested that the mechanism for the switch from mono affinity (KOR) to dual affinity (KOR and MOR) is more complicated than currently thought.

In fact, it is not easy to make a general trend of structure-activity relationship that goes through all the variations of the 6,5-fused rings for each of the opioid receptors. We think it is more suitable and appropriate to put them into groups and make the trends of structure-activity relationship within each group (Figure 3). With non-nitrogenous 6,5fused rings, breaking the trigonal planar geometry of carbon 2 on the 6,5-fused ring, such as in the indane $\mathbf{3}$, diminished the affinity towards KOR (Figure 3A). The indene-2- $\mathbf{2}$ exhibited triple affinity towards KOR, MOR, and DOR, while the indene-3- 1 exhibited dual affinity towards KOR and MOR.

6,5-Fused rings that have an additional heteroatom besides nitrogen, such as $\mathbf{4 ,} \mathbf{6}$, and $\mathbf{1 1}$, displayed significant difference in opioid receptor affinity and selectivity from one another (Figure 3B). While the benzoxazole 4 exhibited no affinity to any of the opioid receptors, the benzimidazole 6 exhibited selective affinity towards MOR and the benzothiazole 11 exhibited selective affinity towards KOR. Meanwhile, the 6,5-fused ring with a nitrogen at the bridge location, indolizine 12, exhibited triple affinity towards KOR, MOR, and DOR. 
A fluorine substitution at the positions 4, 6, and 7 on the indole, such as in the cases of compounds 7, 9, and 10, significantly enhanced the binding affinity to both KOR and MOR (Figure 3C). Meanwhile, a fluorine substitution at the position 5 on the indole, such as in the case of compound $\mathbf{8}$, led to a loss of binding affinity to MOR.

The geometry of carbon 2 on the indole had a significant impact on the binding affinity of the compounds to KOR and MOR. With the trigonal planar geometry of carbon 2 on the indole in the case of salvindolin, MOR affinity was 100 -fold more favorable; meanwhile, breaking the trigonal planar geometry of carbon 2 on the indole, in case of compounds 13 and 14, brought the KOR and MOR affinities to a more even ground (Figure 3D). The $(R)$ indoline isomer 13 had a 14-fold preference towards MOR, while the $(S)$ indoline isomer $\mathbf{1 4}$ had a 2 -fold preference towards KOR.

As mentioned earlier, compounds exhibiting dual agonism to the opioid receptors, such as to MOR and $\mathrm{KOR},{ }^{5,6}$ or to MOR and DOR, ${ }^{7}$ have been suggested to temper undesirable adverse effects while retaining analgesic activity; therefore, we decided to focus our in vivo studies on the compounds that possessed dual or triple activity on the opioid receptors. Some of these compounds had higher binding affinities to a receptor than others but resulted in diminished $\mathrm{EC}_{50}$ values, such as compound $\mathbf{9}$ at KOR and MOR. Meanwhile, some other compounds that had higher binding affinities to a receptor than others retained good $\mathrm{EC}_{50}$ values, such as compound $\mathbf{1 0}$ at KOR and MOR. A higher binding affinity to a receptor does not always translate to a better potency as potency also depends on efficacy, which is the intrinsic activity of the drug-receptor complex to produce a maximum functional response. Therefore, we chose our lead compounds for in vivo studies based on both their dual or triple activities of the compounds and the potencies of compounds at the opioid receptors. Compound $\mathbf{2}$ is one of the only two compounds that had triple activity on KOR, MOR, and DOR (compound $\mathbf{1 2}$ is the other one), but unlike 12, compound 2 retained a good $\mathrm{EC}_{50}$ value at MOR, so $\mathbf{2}$ was chosen for in vivo studies. Compound $\mathbf{1 0}$ is one of a few compounds that had dual activity on KOR and MOR, but unlike others, compound $\mathbf{1 0}$ retained good $\mathrm{EC}_{50}$ values at both KOR and MOR, so $\mathbf{1 0}$ was also chosen for in vivo studies.
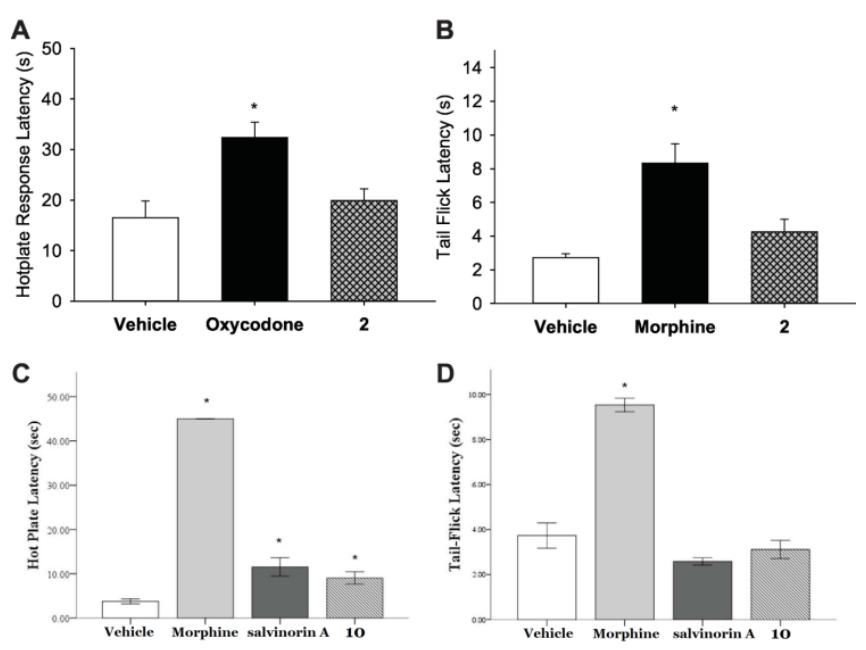

Figure 4: Effects of compounds 2 and 10 in the hot plate and tail flick thermal antinociception assays. Average latency to hind paw lick or flutter in the hot plate assay (A) or latency to remove tail in the tail flick assay (B) following dosing with vehicle $(\mathrm{n}=5), 5 \mathrm{mg} / \mathrm{kg}$ oxycodone $(\mathrm{n}=5), 5 \mathrm{mg} / \mathrm{kg}$ morphine $(\mathrm{n}=5)$, or $2 \mathrm{mg} / \mathrm{kg}$ compound $2(\mathrm{n}=8)$. Similarly, average latency to hind paw lick or flutter in the hot plate assay (C) or latency to remove tail in the tail flick assay (D) following dosing with vehicle $(n=10)$, morphine $(\mathrm{n}=10), \quad 2.5 \mathrm{mg} / \mathrm{kg}$ salvinorin A $(\mathrm{n}=10)$, or $2.5 \mathrm{mg} / \mathrm{kg}$ compound $10(\mathrm{n}=10)$. All data were analyzed with a One-Way ANOVA post-hoc Fisher's LSD, with significant differences denoted * when $\mathrm{p}<0.05$.

The anti-nociceptive effects of the two lead compounds, 2 and 10, against acute pain were assessed in male C57BL/6 mice (Figure 4). In the hot plate assay, which examines supraspinallymediated nociception, mice treated with oxycodone showed a significant increase in hot plate response latencies compared to vehicle treated mice. Mice treated with compound $\mathbf{2}$ did not show an increase in hot plate response latency and were not significantly different than vehicletreated mice. Consistent with these findings, a significant main effect for treatment for was found $(p<0.05)$, prompting post hoc analysis. Pair-wise comparisons on these data compared to vehicle- 
treated mice showed oxycodone produced a significant increase in hot plate response latencies $(p=0.002)$, while 2 did not $(p=0.484)$ (Figure 4A). In the spinally mediated thermal tail-flick nociception assay, mice treated with morphine showed a significant increase in tail-flick latencies while compound $\mathbf{2}$ did not. Consistent with these findings, a significant main effect for treatment for was found $(p<0.05)$, prompting post hoc analysis. Pair-wise comparisons revealed morphine-treated mice had significant higher tailflick withdrawal latencies compared to vehicletreated mice $(p<0.001)$, while withdrawal latencies for compound $\mathbf{2}$ were not statistically significant $(p=0.194)$ (Figure 4B).

A separate cohort of mice was utilized to assess compound 10. Mice treated with morphine and compound $\mathbf{1 0}$ showed a significant increase in hot plate response latencies at 30 minutes posttreatment compared to vehicle-treated mice, but not at 15 minutes post-treatment. Consistent with these findings, a significant main effect for treatment for was found ( $p<0.05)$, prompting post hoc analysis. Pair-wise comparisons revealed significant increase in response latencies in morphine treated mice $(p<0.001)$ (Figure 4C). A significant increase in response latencies in mice treated with $2.5 \mathrm{mg} / \mathrm{kg}$ of compound 10 was observed at 30 minutes $(p<0.05)$ (Figure 4C) but not at 15 minutes post-treatment $(p=0.136)$. These data suggest this antinociceptive effect is not immediate upon administration. The effects of salvinorin $\mathrm{A}$ and compound 10 were not statistically different from each other in either the hot plate or tail-flick assay $(p>0.05)$, as both only exhibited anti-nociceptive effects on the hot plate (Figure 4C-D).

These results highlighted that compound $\mathbf{1 0}$ exerted significant anti-nociceptive effects in the hot plate test but not in the tail-flick test, suggesting the anti-nociceptive effect of $\mathbf{1 0}$ was mediated supraspinally. While the extent of their effects did not meet the level of our controls oxycodone and morphine, the doses of these control compounds were higher, especially when one considers their in vitro affinity and potency. This difference may also be related to the receptor selectivity. We used a smaller dose for compound 10 because its solubility was limited at higher doses in the vehicles traditionally used to administer test compounds to mice. While salvinorin A was soluble at higher doses, we elected to maintain consistent doses of salvinorin $\mathrm{A}$ and compound $\mathbf{1 0}$ to allow for direct comparisons. It is likely that salvinorin A would have had more pronounced effects across all assays at higher doses, as several other publications have indicated pronounced antinociceptive and anxiogenic effects of salvinorin A.
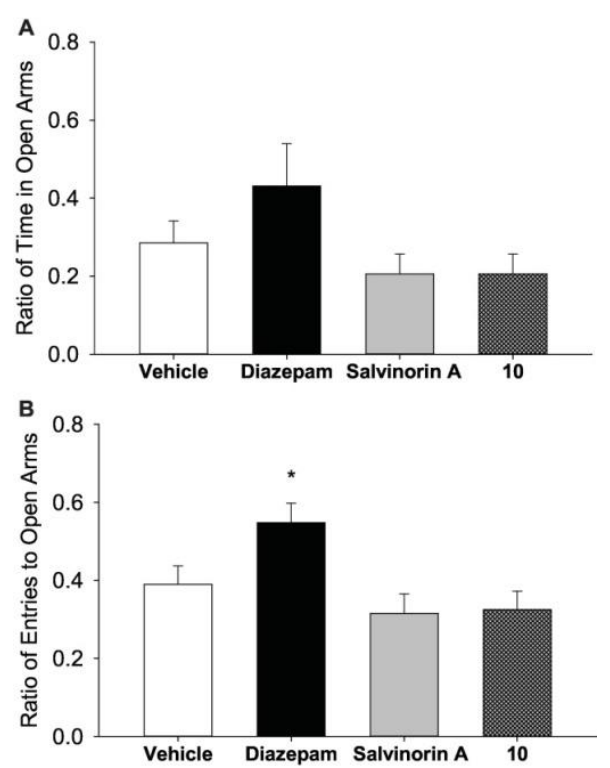

Figure 5: Anxiogenic/Anxiolytic Effects of compound 10 in the elevated plus maze test. A) The average ratio of time spent in the open arms for groups of male mice treated with vehicle $(\mathrm{n}=10), \quad 3 \mathrm{mg} / \mathrm{kg} \quad$ diazepam $\quad(\mathrm{n}=9), \quad 2.5 \mathrm{mg} / \mathrm{kg}$ salvinorin $\mathrm{A}(\mathrm{n}=10)$, and $2.5 \mathrm{mg} / \mathrm{kg}$ compound $\mathbf{1 0}$ $(n=10)$. The ratio was calculated using this formula: total duration in open arms / (total duration in closed + open arms). B) The average ratio of total entrances into open arms for the same groups in A. This ratio was calculated using this formula: total number of entrances into open arms / (total number of entrances into closed + open arms). Means +/- SEM were compared with a One-Way ANOVA post-hoc Dunnett's test vs. vehicle control, with significant differences denoted $*$ when $\mathrm{p}<0.05$. 
The anxiolytic and/or anxiogenic effects of compound $\mathbf{1 0}$ was assessed in the elevated plus maze 30 minutes following intraperitoneal administration. No significant differences were observed in mice treated with compound $\mathbf{1 0}$ in the cumulative duration spent in the open arms, the ratio of time spent in the open arms, or the ratio of total entrances into the open arms (Figure 5A-B) ( $\mathrm{p}=0.82,0.74$, and 0.34 respectively). Diazepam control significantly increased the percent of entrances into the open arms $(\mathrm{p}=0.034)$; however, this dose of diazepam was sedative because a significant reduction in the overall locomotor activity and velocity of movements in the maze was observed (both $\mathrm{p}<0.001$; Supporting Information Table 1S). No differences in any of these parameters were noted with compound $\mathbf{1 0}$ or salvinorin $A(p=0.22$ and $0.66, p=0.43$ and 0.34 , respectively).

These results highlighted that compound $\mathbf{1 0}$ did not cause anxiogenic or anxiolytic effects in the elevated plus maze, thus providing further strong evidence that dual KOR and MOR agonists can temper undesirable adverse effects while retaining analgesic activity. One limitation of our in vivo studies was the exclusion of female cohorts of mice. As sex-specific differences in nociceptive assays have been reported, it would be important to include sex as a biological variable in follow-up studies related to salvinorinbased compounds.

We have reported an introduction of various 6,5-fused rings to $\mathrm{C} 2$ of the salvinorin scaffold via an ester linker. The compounds were subjected to in vitro evaluation to determine their affinity, efficacy, and functionality on classical opioid receptors. The results showed that some of these compounds have dual agonism on KOR and MOR, while some have triple agonism on KOR, MOR, and DOR. The compounds were categorized into groups and the trends of structure-activity relationship within each group were identified. The trends disproved two current hypotheses on the structure-activity relationship of salvinorinbased, dual KOR and MOR agonists. As selective $\mathrm{mu}$ or kappa receptor agonists often cause harmful side effects such as addiction, dysphoria, reduced bowel motility, tolerance, convulsions, and overdose, and dual agonism to the opioid receptors, such as to MOR and KOR, or to MOR and DOR, have been suggested to temper undesirable adverse effects while retaining analgesic activity, two lead compounds, $\mathbf{2}$ and $\mathbf{1 0}$, which possessed triple agonism on KOR, MOR, and DOR, and dual agonism on KOR and MOR, respectively, were selected for in vivo studies. The results showed that $\mathbf{1 0}$ exerted significant antinociceptive effects in the hot plate test but not in the tail-flick test, suggesting the anti-nociceptive effect of $\mathbf{1 0}$ was mediated supraspinally. The antinociceptive effect of $\mathbf{1 0}$ was not immediate upon administration. In addition, compound $\mathbf{1 0}$ did not cause anxiogenic or anxiolytic effects in the elevated plus maze, thus providing further strong evidence that dual KOR and MOR agonists have therapeutic advantages over selective opioid receptor agonists.

\section{SUPPLAMENTARY MATERIAL}

The Supporting Information is available free of charge at: https://pubs.acs.org/

Materials and methods, and characterization of the synthesized compounds

\section{AUTHOR INFORMATION}

\section{Corresponding Author}

* hle@olemiss.edu

\section{AUTHOR CONTRIBUTIONS}

N.S.A. synthesized compounds 1 and 3-14. A.W.K. synthesized compound 2. N.S.A. and N.M. carried out in vitro studies. H.M.H and N.M.A carried out in vivo studies. S.J.K. contributed in the purification and characterization of the compounds. N.D. and S.M. identified the vehicle used for in vivo studies. J.K.Z contributed as a consultant of the project. H.V.L. designed the project and the compounds and supervised the overall coordination of the research. N.S.A, N.M.A., and H.V.L. wrote the manuscript. All authors approved the final version.

\section{CONFLICT OF INTEREST}

The authors report no conflict of interest. 


\section{ACKNOWLEDGMENTS}

Work was supported by National Institute of General Medical Sciences (P30GM122733 pilot project award to $\mathrm{H}$. V. L. and the Neuropharmacology Core Facility at the University of Mississippi, School of Pharmacy) and funds from the Department of BioMolecular Sciences at the University of Mississippi, School of Pharmacy. The content is solely the responsibility of the authors and does not necessarily represent the official views of these funders.

\section{REFERENCES}

(4) Vowles, K. E.; McEntee, M. L.; Julnes, P. S.; Frohe, T.; Ney, J. P.; van der Goes, D. N. Rates of Opioid Misuse, Abuse, and Addiction in Chronic Pain. Pain 2015, 156 (4), 569-576.

(5) Sałaga, M.; Polepally, P. R.; Sobczak, M.; Grzywacz, D.; Kamysz, W.; Sibaev, A.; Storr, M.; Do Rego, J. C.; Zjawiony, J. K.; Fichna, J. Novel Orally Available Salvinorin A Analog PR-38 Inhibits Gastrointestinal Motility and Reduces Abdominal Pain in Mouse Models Mimicking Irritable Bowel Syndrome. $J$. Pharmacol. Exp. Ther. 2014, 350 (1), 6978.

(6) Keasling, A. W.; Pandey, P.; Doerksen, R. J.; Pedrino, G. R.; Costa, E. A.; da Cunha, L. C.; Zjawiony, J. K.; Fajemiroye, J. O.
Salvindolin Elicits Opioid System-

Mediated Antinociceptive and Antidepressant-like Activities. $J$. Psychopharmacol. 2019, 33 (7), 865-881.

(7) Podolsky, A. T.; Sandweiss, A.; Hu, J.; Bilsky, E. J.; Cain, J. P.; Kumirov, V. K.; Lee, Y. S.; Hruby, V. J.; Vardanyan, R. S.; Vanderah, T. W. Novel Fentanyl-Based Dual $\mu / \delta$-Opioid Agonists for the Treatment of Acute and Chronic Pain. Life Sci. 2013, 93 (25-26), 1010-1016.

Roth, B. L.; Baner, K.; Westkaemper, R.; Siebert, D.; Rice, K. C.; Steinberg, S.; Ernsberger, P.; Rothman, R. B. Salvinorin A: A Potent Naturally Occurring Nonnitrogenous Opioid Selective Agonist. Proc. Natl. Acad. Sci. 2002, 99 (18), 11934-11939.

Johnson, M. W.; MacLean, K. A.; Reissig, C. J.; Prisinzano, T. E.; Griffiths, R. R. Human Psychopharmacology and DoseEffects of Salvinorin A, a Kappa Opioid Agonist Hallucinogen Present in the Plant Salvia Divinorum. Drug Alcohol Depend. 2011, 115 (1-2), 150-155.

(10) Appel, J.; Kim-Appel, D. The Rise of a New Psychoactive Agent: Salvia Divinorum. Int. J. Ment. Health Addict. 2007, 5 (3), 248-253.

Prisinzano, T. E. Psychopharmacology of the Hallucinogenic Sage Salvia Divinorum. In Life Sciences; Life Sci, 2005; Vol. 78, pp 527-531.

(12) Roach, J. J.; Shenvi, R. A. A Review of Salvinorin Analogs and Their KappaOpioid Receptor Activity. Bioorg. Med. Chem. Lett. 2018, 28 (9), 1436-1445.

Kahne, D.; Kerns, R.; Fkuzawa, S.; Ge, M.; Thompson, C. Glyocpeptide Antibiotics Combinational Libraries of Glycopeptide Antibiotics and Methods of Producing Same, 2008.

(14) Ghonim, A. E.; Ligresti, A.; Rabbito, A.; Mahmoud, A. M.; Di Marzo, V.; Osman, N. A.; Abadi, A. H. Structure-Activity Relationships of Thiazole and Benzothiazole Derivatives as Selective 
Cannabinoid CB2 Agonists with in Vivo Anti-Inflammatory Properties. Eur. J. Med. Chem. 2019, 180, 154-170.

(15) Bhat, M.; Belagali, S. L.; Hemanth Kumar, N. K.; Mahadeva Kumar, S. Synthesis and Characterization of Novel Benzothiazole Amide Derivatives and Screening as Possible Antimitotic and Antimicrobial Agents. Res. Chem. Intermed. 2017, 43 (1), 361-378.

(16) Xue, Y.; Tang, J.; Ma, X.; Li, Q.; Xie, B.; Hao, Y.; Jin, H.; Wang, K.; Zhang, G.; Zhang, L.; Zhang, L. Synthesis and Biological Activities of Indolizine Derivatives as Alpha-7 NAChR Agonists. Eur. J. Med. Chem. 2016, 115, 94-108.

(17) Jørgensen, M. R.; Olsen, C. A.; Mellor, I. R.; Usherwood, P. N. R.; Witt, M.; Franzyk, H.; Jaroszewski, J. W. The Effects of Conformational Constraints and Steric Bulk in the Amino Acid Moiety of Philanthotoxins on AMPAR Antagonism. $J$.
Med. Chem. 2005, 48 (1), 56-70.

(18) Giacometti, R. D.; Duchek, J.; Werner, L.; Husni, A. S.; McCurdy, C. R.; Cutler, S. J.; Cox, D. P.; Hudlicky, T. Heteroatom Analogues of Hydrocodone: Synthesis and Biological Activity. J. Org. Chem. 2013, 78 (7), 2914-2925.

(19) Beguin, C.; Richards, M. R.; Li, J.-G.; Wang, Y.; Xu, W.; Liu-Chen, L.-Y.; Carlezon, W. A.; Cohen, B. M. Synthesis and in Vitro Evaluation of Salvinorin A Analogues: Effect of Configuration at C(2) and Substitution at C(18). Bioorg. Med. Chem. Lett. 2006, 16 (17), 4679-4685.

(20) Harding, W. W.; Tidgewell, K.; Byrd, N.; Cobb, H.; Dersch, C. M.; Butelman, E. R.; Rothman, R. B.; Prisinzano, T. E. Neoclerodane Diterpenes as a Novel Scaffold for $\mu$ Opioid Receptor Ligands $\uparrow$. J. Med. Chem. 2005, 48 (15), 4765-4771. 
TABLE OF CONTENT

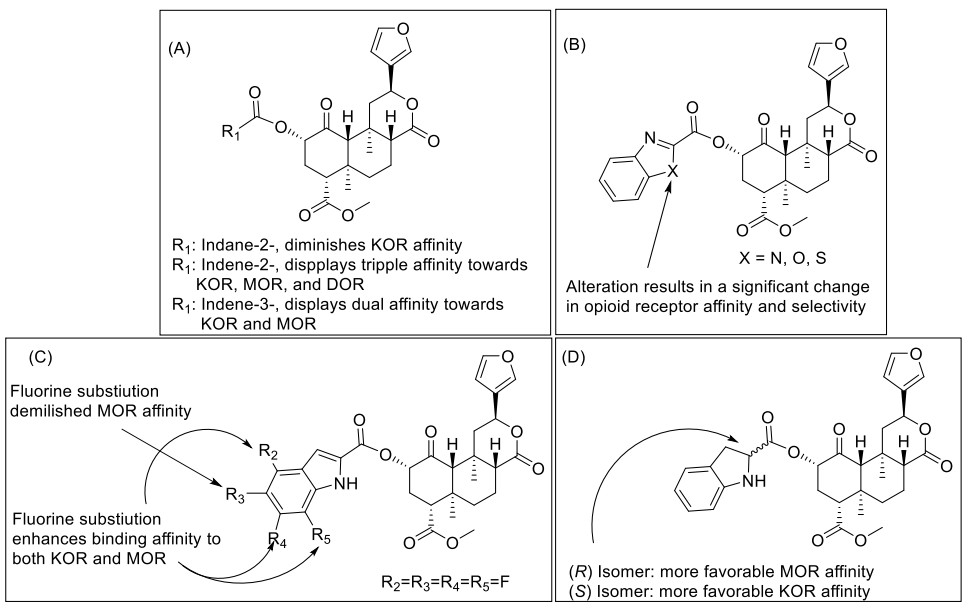

